\title{
Treatment of Impetigo with Antiseptics- Replacing Antibiotics (TIARA) Trial- a single blind randomised controlled trial in school health clinics within socioeconomically disadvantaged communities in New Zealand
}

\section{Sarah Primhak}

The University of Auckland https://orcid.org/0000-0002-6464-5217

\section{Alicia Gataua}

National Hauora Coalition

\section{Diana Purvis}

Starship Children's Health

John MD Thompson

The University of Auckland School of Medicine

\section{Cameron Walker}

The University of Auckland

Emma Best ( $\square$ e.best@auckland.ac.nz )

The University of Auckland

\section{Alison Leversha}

Starship Children's Health

\section{Research Article}

Keywords: impetigo, topical, antimicrobial, resistance, treatments, trial

Posted Date: November 8th, 2021

DOI: https://doi.org/10.21203/rs.3.rs-271692/v1

License: (1) (i) This work is licensed under a Creative Commons Attribution 4.0 International License. Read Full License

Version of Record: A version of this preprint was published at Trials on February $2 \mathrm{nd}, 2022$. See the published version at https://doi.org/10.1186/s13063-022-06042-0. 


\section{Abstract}

\section{Background}

Impetigo is a common and contagious bacterial skin infection, affecting children worldwide but it is particularly prevalent in deprived communities. In New Zealand widespread prescribing of the topical antibiotic fusidic acid had led to an increase in antimicrobial resistance of Staphylococcus aureus. Alternative treatments are urgently being sought and as impetigo is a superficial infection, it has been suggested that topical antiseptics such as hydrogen peroxide or simple wound care alone may treat impetigo while avoiding the risk of increased antimicrobial resistance.

Methods

This protocol for a non-inferiority, single-blind randomised controlled trial compares topical fusidic acid with topical hydrogen peroxide and with simple wound care in the treatment of childhood impetigo. Participants are randomised to one of the three treatments for five days and the primary outcome is clinical improvement. This is assessed through paired photographs analysed by graders blinded to treatment arm. The trial is based in school health clinics in an urban centre in New Zealand. Comparison of antimicrobial resistance patterns pre- and post-treatment is also performed.

Discussion

Special note is made of the need to involve the communities most affected by impetigo in the design and implementation of the clinical trial in order to recruit the children most in need of safe and effective treatments.

Trial registration: Australian New Zealand Clinical Trials Registry (ANZCTR) 12616000356460

\section{Introduction}

Impetigo is a contagious superficial bacterial skin infection, predominantly affecting children. ${ }^{1,2}$ It is described as one of the top 50 most common diseases, ${ }^{3}$ with a global prevalence estimated at 162 million. ${ }^{4}$ New Zealand (NZ) is a high-income country in the South Pacific with a diverse population including tangata whenua- NZ indigenous population (Māori) who make up $17 \%$ of the population. During western colonisation NZ established the Treaty of Waitang $\mathrm{i}^{16}$ between the Crown and Māori which ensures a legal requirement to good governance and the right to equitable health outcomes for indigenous people. ${ }^{16}$ While the Pacific peoples of NZ (those from the Polynesian and Melanesian Pacific islands surrounding NZ) are not part of the treaty agreement, they make up a significant proportion of NZ's population ( 7\%) and there is recognition of significant inequities for both Māori and Pacific populations and the obligation to address these. Primary care is generally funded for children under 13 years of age but socioeconomic barriers to care are still recognised. NZ experiences a particularly high burden of skin and soft tissue infections with as many as $11 \%$ of NZ children aged under 15 years 
consulting their primary care provider for skin infections annually. There is an inequity in the burden of disease with the highest rates seen in children of Māori and Pacific peoples.

As a superficial infection, impetigo is frequently treated topically rather than systemically. Since the early 2000s New Zealand has experienced high prescribing rates of the topical antibiotic fusidic acid; rates of dispensing are highest for preschool children, followed by those age $75+$ and $5-14$ years. Dispensing is also highest in Pacific Island and Māori ethnicities. ${ }^{6,7}$ Following the increase in prescribing, a subsequent rise in fusidic acid resistant $S$. aureus isolates has been reported, resistance is now demonstrated in $28 \%$ of NZ S. aureus isolates. ${ }^{8}$ In 2005, a fusidic acid resistant and methicillin resistant $S$. aureus (MRSA) clone was identified and has become the dominant MRSA clone in New Zealand. ${ }^{9}$ This suggests that widespread community use of fusidic acid has not only led to selection of fusidic acid resistant clones but also concurrent MRSA. In an attempt to limit the development of further antimicrobial resistance by avoidance of topical antibiotics, NZ has updated national impetigo guidance. While oral antibiotics remain the first line for severe or multi-lesional impetigo, in mild-to-moderate disease topical fusidic acid is no longer first line; superseded by the advice to use an antiseptic cream; $1 \%$ hydrogen peroxide. ${ }^{10}$ The UK National Institute of Clinical Excellence (NICE) have also released a draft guideline on impetigo proposing the same change. ${ }^{11}$ These prescribing guidelines are based on evidence from a single randomised controlled trial performed in 1993 with no information provided on fusidic acid resistance rates within the study population. This demonstrated "a tendency towards somewhat lower efficacy in [hydrogen peroxide] compared to [fusidic acid]" with no significant difference between the groups. ${ }^{12}$ Since that time antimicrobial resistance patterns to fusidic acid have changed within NZ. ${ }^{6,7}$ and potentially within other countries where it is used topically or systemically.

In the past clinical trials of impetigo have presented challenges and this trial protocol seeks to address these. Impetigo is predominantly a disease seen in the community and it is thought that primary care presentations represent only a small proportion of cases. Many families never seek medical review, this is particularly relevant in a population where financial concerns have been shown to limit health seeking behaviours. ${ }^{13} \mathrm{~A}$ clinical impetigo trial should therefore, ideally be based within a community setting. Impetigo is a disease of childhood, a challenging group to enrol in clinical trials with previous trials on impetigo treatment including large numbers of adults; the RCT upon which current practice is based, recruited patients with a mean age of 17 years. ${ }^{12}$

Defining primary outcomes in impetigo treatment is also challenging as demonstrated by the variety of outcomes measures across prior clinical trials. A systematic review concluded that more robust outcome measures were required. ${ }^{14}$ Time to reported clinical endpoints have ranged between 1 to 3 weeks after starting treatment. Some trials have used complete cure while others are satisfied with clinical improvement of varying definitions. Many are based on observer defined definitions but even the more objective scores such as the Skin Infection Rating Score (SIRS), have been used as a primary outcome measure in a variety of ways; including an absolute reduction in score, predefined absolute decrease in score or as a percentage decrease from the baseline. 
To best inform our practice, relevant to the groups most affected by impetigo in NZ, our trial requires a majority recruitment of children of Māori and Pacific children. There are marked inequities in health outcomes for Māori and Pacific island children living in New Zealand and it is critical that health research is focused on understanding and addressing these inequities. Impetigo is contributing to inequity in health outcomes and therefore is crucially important to Māori and Pacific health. With the burden of skin disease affecting predominately Māori and Pacific children appropriate cultural engagement with these communities was recognised as a key factor, both for successful trial completion as well as ensuring meaningful results which will be acceptable, generalisable, and implementable for those most affected by impetigo.

\section{Objectives}

We hypothesize both simple wound care and topical hydrogen peroxide are non-inferior to topical fusidic acid in the treatment of impetigo.

Therefore, the aims of this clinical trial are:

1. To compare the effectiveness of topical fusidic acid with topical hydrogen peroxide and simple wound care in the treatment of mild-to-moderate impetigo in a community with both high rates of impetigo and increasing fusidic acid resistance.

2 ) To examine potential changes in the antimicrobial resistance of skin pathogens in response to these different treatments for impetigo.

Trial design: TIARA is an open label, single-blind, non-inferiority randomised controlled trial with three parallel treatment groups. The primary endpoint is clinical improvement at seven days. Randomisation is performed 1:1:1 within each school clinic.

\section{Methods: Participants, Interventions, And Outcomes}

Study setting: Auckland city has a temperate climate and is a large urban centre of 1.6 million people. Primary school health clinics serve the more socioeconomically disadvantaged areas within two of the three district health boards in the Auckland region: Auckland and Counties Manukau. These health clinics provide primary care, including free skin and throat infection management, to students aged 5-13 years. ${ }^{17,18}$ The school nurses running the clinics are invited to participate in this study to provide a potential eligible population of $\sim 10,000$ enrolled students. Due to their over representation of socioeconomic disadvantage, over $90 \%$ of children in the schools are of Māori or Pacific island ethnicity and there is a high rate of impetigo.

\section{Eligibility Criteria}


Children meeting the eligibility criteria are identified and their caregivers contacted to explain the study and obtain consent. Caregivers must provide verbal informed consent before any study procedures occur. This is then followed by written informed consent (see Appendix 1 for sample of Informed Consent Form).

\section{Inclusion criteria:}

Children eligible for the trial must comply with all of the following prior to randomisation:

1. Enrolled in one of the participating school clinics

2. Mild-to-moderate impetigo

3. Aged $5-13$ years.

Exclusion criteria:

1. Severe impetigo requiring oral antibiotics; defined as extensive lesions ( $>3$ lesions or $>5 \%$ body surface area), presence of cellulitis or fever $>38.5 \mathrm{C}$

2. Children who are immunocompromised

3. Known allergy to study drugs

4. Current use, or use within the previous 5 days, of topical or oral antimicrobials

5. Commencement of antimicrobials for other reasons during the trial period

6. Failure to obtain informed consent for randomisation or withdrawal of consent

Excluded children continue with treatment according to the existing school health clinic standard operating procedures (SOP).

\section{Intervention}

All lesions are cleaned with saline and scabs gently removed. For the group randomised to fusidic acid, $2 \%$ fusidic acid ointment (DP Fusidic Acid, Douglas Pharmaceuticals Ltd, Auckland, NZ) is applied topically and for the hydrogen peroxide arm, $1 \%$ hydrogen peroxide cream (Crystaderm, AFT Pharmaceuticals, Auckland, NZ) is applied topically. In both cases an adequate amount to cover each lesion is used and dressing(s) then applied. A tube of appropriate topical medication is supplied for the child and/or caregivers to continue applications twice daily for 5 days with dressing changes.

Participants allocated to simple hygiene measures receive no medication but a dressing is applied following the cleaning of the lesion(s). All participants are provided with supplies to allow them to clean and redress the wound twice daily for 5 days. Low adherent wound pads are used so as not to interfere with the wound healing process. In all groups, scabies is treated if present.

\section{Modifications}

All adverse effects, including pain, itch or allergy to study medication, will be reported; the study medication must be withdrawn, and the patient changed to routine treatment as per the SOP of the health 
clinic. If clinical deterioration while on study medication is identified by the school nurse, the study medication may be withdrawn at the nurse's discretion. In this case the patient will be changed to routine treatment as per the standard operating procedures of the health clinic.

\section{Adherence}

Face to face reminders of adherence are provided by nurses at both day 0 and day 2 visits and adherence over the trial period will be assessed on days 2 and 7. Sticker charts are provided for each participant to encourage adherence.

\section{Participant Timeline}

On the first visit (day 0) demographic data, inclusion and exclusion criteria and verbal consent are obtained. All lesions are cleaned and the single largest lesion is photographed using a digital camera and a bacteriological swab is taken from the same lesion as the photograph. The patient is then randomised and the appropriate treatment is commenced and continued for five days. Two days after enrolment into the trial (day 2), a safety check is performed by the school nurse to assess for rapid worsening of the impetigo or for adverse effects. The safety check can be performed between days $2-4$ if necessary to allow for day 2 falling on a weekend. Seven days after commencing the trial (day 7), the participant is reassessed by the nurse. A second set of photographs and repeat bacterial swab is taken from the same lesion as originally documented. Both child and caregiver complete a verbal questionnaire. Caregivers can withdraw a participant from the study at any stage.

\section{Sample size}

On the assumption of non-inferiority between hygiene measures and topical fusidic acid and between topical hydrogen peroxide and fusidic acid, and a predicted efficacy of fusidic acid of $80 \%$; we require 160 patients in each group. This provides $80 \%$ power and a one-sided a of 0.05 to show non-inferiority $(10 \%$ margin) between each group and topical fusidic acid. In order to allow for $10 \%$ loss to follow up and subsequent exclusions, recruitment of 178 participants to each group is required. Subsequent exclusions and loss to follow up are defined as participants not available for follow up at day 7 or when two digital images are not available to assess.

\section{Recruitment}

School health teams within recruiting schools will follow existing SOPs. Community health workers attend every class 3-5 days each week during the school term and ask children to self-identify any skin infections. These children are then reviewed by the school nurse. If the lesions are confirmed as impetigo then eligibility for participation is assessed.

\section{Allocation concealment mechanisms and implementation}


Randomisation is implemented by block randomisation within each participating school, sequence allocation code was written in . $^{19}$ Children are randomly allocated (1:1:1) to topical fusidic acid, topical hydrogen peroxide or simple hygiene measures. Participating schools are randomised separately to limit bias caused by excess recruitment to any individual arm within a single school. Allocation is performed by school nurses using the pre-generated codes contained in sealed, opaque, sequentially numbered envelopes. Both the participant and school nurse are aware of treatment allocation due to the appearance of the study medications (e.g. hydrogen peroxide has a silvery sheen) or lack of study medication in the simple hygiene measure arm. However, investigators, photograph reviewers and laboratory staff are blinded to allocation.

\section{Data Collection Methods}

\section{Demographic characteristics}

Baseline characteristics of participants are collected at the time of randomisation and reported per randomisation group. The following demographic characteristics will be reported: age, gender, ethnicity, weight, history of pre-existing skin disease, allergies, and location of most severe lesion.

\section{Primary outcome methods}

The primary outcome of this trial is treatment success assessed by comparison of digital photographs taken at day 0 and 7 or clinical deterioration based either on digital images or on nursing assessment leading to discontinuing trial medication if second image is not available. To aid in standardisation, the primary outcome uses digital photographs that can be assessed retrospectively by blinded reviewers. The single largest lesion is photographed prior to commencing treatment. Using a digital camera at a distance of $15 \mathrm{~cm}$ with an adhesive paper tape measure and unique study ID placed next to the lesion. Three digital images are taken at each data collection visit and all are submitted to the study investigators. The single best quality image is then selected by the lead investigator (SP) for outcome assessment. Pairs of images are presented to three individual assessors who are blinded to intervention arm. The method of image presentation is randomised such that assessors are unaware of which image was taken first and which second. The outcome will be treatment success if the images are considered healed or improved, and treatment failure if they are the same or worse or if the reviewers cannot determine the outcome based on the digital images. Where there is discordance between assessor opinions, the majority opinion of two of three reviewers will be considered the correct assessment. Patients removed from the trial protocol by school nurses or general practitioners before seven days due to clinical deterioration will also be considered treatment failures. This process has been standardised previously in another large RCT of treatment of impetigo in remote access locations; different from our multicentre urban setting. ${ }^{20}$

\section{Clinical assessments}

Clinical assessments are standardised and recorded on the existing skin assessment database used for school health clinics. A written description of the location of the lesions and identification of the primary 
lesion being used for assessment is recorded. A paper template is also provided to allow nurses the option to mark on a diagram the location of the lesions.

Seven days after commencing the trial (day 7), the participant is re-assessed by the school nurse, who also records whether the lesion has improved. Both child and caregiver are asked to comment on their satisfaction with treatment and any adverse events related to the medication, including itch, pain or allergy and school records are checked for absence over the prior seven days.

\section{Microbiology}

All children have a bacteriological dry cotton swab taken from the most severe lesion at presentation and at day 7. Swabs are cultured onto blood agar and any clinically significant growth is reported. European Committee on Antimicrobial Susceptibility Testing (EUCAST) susceptibility method and criteria are used for $S$. aureus susceptibility testing. Susceptibility is tested to commonly used skin and soft tissue antimicrobials; fusidic acid, flucloxacillin, erythromycin, clindamycin and co-trimoxazole. If MRSA is identified then extended susceptibility is performed, including mupirocin and tetracyclines. No susceptibility testing is performed on $S$. pyogenes.

\section{Outcomes}

\section{Primary Outcome:}

The primary outcomes of this trial is treatment success assessed by comparison of digital photographs or clinical deterioration based either on digital images or on nursing assessment leading to discontinuing trial medication.

\section{Secondary outcomes}

- Clinical success will be compared between group. This will be defined by

1. nursing opinion that the impetigo has improved at day seven

2. participant and/or caregiver opinion that the impetigo has improved at day seven

- Microbiological secondary outcomes are eradication of S. pyogenes and/or S. aureus on day 7 and development of antibiotic resistance on day seven compared to baseline

- Educational impact is assessed by comparison of school absence over the seven days of the trial period

- Adverse events will be compared across study arms.

\section{Retention}

On successful completion of the trial protocol a supermarket voucher worth NZ\$20 will be offered to the family as koha; a thank you gift. 


\section{Data Management}

Trial data is stored using a study identification number on a password protected access database maintained on a secure network. This database is also used to randomise images and presents anonymised pairs of photographs to the graders for analysis, and records the outcomes of the grading.

\section{Statistical analyses}

For baseline data, dichotomous variables will be summarised as proportions of patients in each treatment group, differences between groups will be assessed using a Chi-square statistic, and where small cell sizes (less than 5) are present, a Fisher's exact test will be used. Continuous variables with an underlying normal distribution will be summarised as mean and standard deviation and differences between groups will be assessed using a Students t-test. Otherwise distributions will be either transformed if suitable and t-tests performed with reporting of geometric means. Otherwise distributions will be reported as median and interquartile range and differences in groups assessed using a Wilcoxon rank non-parametric tests with Hodges-Lehmann estimates and 95\% confidence intervals.

Analysis will be performed after completion of recruitment. Both hydrogen peroxide and simple hygiene groups will be compared independently with fusidic acid with $95 \%$ confidence interval. Non-inferiority will be defined as a treatment success rate of no more than $10 \%$ below that of the fusidic acid success rate. An intention to treat and per protocol analysis will be performed using all patients with available primary outcome data. Patients without primary outcome data or for whom caregivers withdrew consent to participate will not be included in the analysis.

To investigate predictors of treatment success, backwards stepwise random-effects logistic regression will be performed on a priori and other variables identified as different in baseline characteristics between randomisation groups.

\section{Data Monitoring}

A Data Safety Monitoring Board (DSMB) has been convened. An interim safety analysis will be performed after recruitment of 150 participants. The DSMB will have unblinded access to all data and will discuss the outcome of the analysis with the trial steering committee. The unblinded data, apart from the outcome of the DSMB decision, will not be made available to the authors prior to the completion of the trial and unblinding.

\section{Harms}

Any adverse event will be reported; these are defined as any untoward medical occurrence in a subject without regard to the possibility of a causal relationship after entry into the study and until the completion of the study. At day two and day seven of the trial, a safety check is performed by the school nurse to assess for rapid worsening of the impetigo or for adverse effects. If these occur then the participant can be withdrawn from the trial at the discretion of the nurse. 


\section{Auditing}

Regular visual review of the data will be performed by the lead investigator (SP) for completeness and quality of the data.

\section{Discussion}

The aim of this study is to investigate the relative effectiveness of non-antibiotic management of impetigo in children. The utilisation of existing school health clinics located within the more socioeconomically deprived communities in Auckland, NZ, allows access to a large population of children within a community who might not otherwise seek medical help. This allows access to an appropriate cohort of children at high risk of impetigo.

Engaging authentically with Māori and Pacific communities is critical to the success of this project. As outlined above Māori and Pacific communities living in New Zealand face a number of barriers to accessing appropriate and timely primary healthcare for potentially preventable conditions such as impetigo. ${ }^{13}$ Research is critical to address these inequities and ideally should be undertaken using a partnership approach with Māori and Pacific researchers, in a culturally appropriate way. The crucial resource in engaging with the community were the community representative school nurses, already known and trusted by the families of potential participants.

The National Hauora Coalition (NHC), a Māori primary health organisation, were consulted early in study design and a Māori nurse leader (AG) managed key implementation aspects of the trial and engendered the support of the school nurses who represent both Māori and Pacific Island healthcare workers. Information leaflets have been provided in multiple languages, including Te Reo Māori, Tongan and Samoan, and nursing staff provide additional verbal information as a more acceptable communication.

${ }^{13}$ Specific acts of recognition and practical reimbursements have been shown to contribute significantly to a sense of value and reduce the financial strain when accessing healthcare. ${ }^{13}$ To acknowledge this a gift (koha) of a NZ\$20 supermarket voucher is offered to each family after completion of the trial.

Because of the large and disparate pool of recruiters it is vital that the primary outcome is as comparable and unbiased as possible. Digital images allow for centralised assessment despite the distance between recruiting sites. However, this means the existing scoring system (SIRS) is not practical as variable included in the scoring such as warmth, pain and itch are not amenable to visual assessment alone. For this reason, assessor defined scoring was used. Erythema is also one of the elements of the SIRS scoring system and when assessing children with darker skin, this is often underestimated, ${ }^{21}$ leading to minimisation of the severity of lesions in those with darker skin. The use of digital images and multiple reviewers for the primary outcome aims to minimise the bias inherent in the majority of previous studies on impetigo and provide a reproducible outcome, consistent with real world improvement. This remains an imperfect measure as it is dependent on good quality digital images and reviewer defined assessment. Due to the visible difference in the topical medications, neither school nurses nor participants could be 
blinded to treatment. This introduces a potential element of bias for those removed from the study early due to clinical treatment failure. It was considered unfeasible to introduce a placebo medication to the simple wound hygiene group. Potentially any ointment without antimicrobial properties could provide an environment for bacterial growth or negatively affect bacterial growth, potentially changing the outcome for this group.

Topical antibiotics remain the mainstay of treatment for mild-to-moderate impetigo in many countries around the world, including New Zealand. Widespread community use and a tendency for prolonged courses, mean that they excel at inducing antimicrobial resistance. Evidence for antiseptic use or simple hygiene measures in impetigo is extremely limited: this will be only the second trial to compare topical antiseptics and antibiotics and the first to take current antimicrobial resistance patterns into account. On the background of a worldwide increase in antimicrobial resistance and with increasing recognition of the importance of antimicrobial stewardship, it is timely to re-address the evidence for the role of antiseptics and antibiotics in the treatment of impetigo. This is only possible however, if the most affected communities are appropriately engaged in this research.

Trial status: this trial is completing initial recruitment. Follow up data is continuing to be collected.

\section{Declarations}

Ethics approval and consent to participate:

This study has been approved by the Northern A Committee of the Human and Disability Ethics Committee of New Zealand (HDEC) 16/NTA/113/AM03. Locality approval has been obtained from the research offices of both Auckland and Counties Manukau District Health Boards and Māori research approvals obtained at each locality.

Verbal consent from the caregiver is obtained on an individual basis at the point of identification of impetigo and then followed with written informed consent. Children older than 7 years of age have the opportunity to sign an assent form. Consent is obtained by the school nursing staff after training by the research trial team.

Consent for publication: No patient-identifiable information will be published.

Availability of data and materials: Patient consent/ assent form is attached. Materials including patient information and data collection sheets are available on request from the authors.

Competing interests: None

Funding: The TIARA Trial is funded by a Cure Kids New Zealand Project Grant: Interventional Research Focused on Addressing Child Health Conditions Associated with Deprivation (ref. 4000). Auckland DHB Charitable Trust (A+ Trust) Research Grant (ref. 9664) and Tupu Research Grant, Ko Awatea provided the salaries for the research nurses. The New Zealand Society Wound Care Grant funded the cameras for the 
trial and the Joan Mary Reynolds Charitable Trust and the Starship Foundation provided funding for SP's salary. The design, management, analysis and reporting of the study are entirely independent of the manufacturers of the medications involved and of all funders.

Authors contributions: EB, AL, DP and DL conceived of the study. SP, EB, AL and DL initiated the study design and $A G$ enabled implementation, site co-ordination and cultural consultation. AL, PA, EB and SP are grant holders. CW and JT provided statistical expertise in clinical trial design. All authors contributed to refinement of the study protocol and approved the final manuscript.

Sponsor: University of Auckland, Auckland, New Zealand.

Acknowledgements: With thanks to Diana Lennon, Deborah Williamson, Helen Gordon and Philippa Anderson.

\section{References}

1. Baltimore RS. Treatment of impetigo: A review. PIDJ. 1985;4(5):597-601.

2. Koning S, van der Sande R, Verhagen AP, et al. Interventions for impetigo. Cochrane Database of Systematic Review. 2012;1(1).

3. Hay RJ, Johns NE, Williams HC, et al. The global burden of skin disease in 2010: An analysis of the prevalence and impact of skin conditions. Journal of Investigative Dermatology. 2014;134(6):152734.

4. Bowen AC, Mahé A, Hay RJ, et al. The global epidemiology of impetigo: A systematic review of the population prevalence of impetigo and pyoderma. PloS one. 2015;10(8):e0136789.

5. O'Sullivan C, Baker MG. Skin Infections in Children in a New Zealand Primary Care Setting: Exploring beneath the tip of the iceberg. N Z Med J. 2012;125(1351):70-9.

6. Williamson DA, Ritchie SR, Best E, Upton A, Leversha A. A Bug in the Ointment- Topical Antimicrobial Usage and Resistance in New Zealand. The New Zealand Medical Journal. 2015;128(1428):103-9.

7. Vogel A, Lennon D, Best E, Leversha A. Where to from here? the treatment of impetigo in children as resistance to fusidic acid emerges. The New Zealand Medica Journal. 2016;129(1443):77.

8. Williamson DA, Monecke $\mathrm{S}$, Heffernan $\mathrm{H}$, et al. A cautionary tale: High usage of topical fusidic acid and rapid clonal expansion of fusidic acid-resistant staphylococcus aureus. Clin Infect Dis. 2014;59(10):1451-4.

9. Baines SL, Howden BP, Heffernan $\mathrm{H}$, et al. Rapid emergence and evolution of staphylococcus aureus clones harboring fusC-containing staphylococcal cassette chromosome elements. Antimicrob Agents Chemother. 2016;60(4):2359-65.

10. Best practice advocacy centre (BPAC) guideline. https://bpac.org.nz/antibiotics/guide.aspx. Updated 2018. 
11. National Institute of Clinical Excellence. Draft NICE guideline. impetigo: Antimicrobial Prescribing; 2019.

12. Christensen $\mathrm{OB}$, Anehus S. Hydrogen peroxide cream: An alternative to topical antibiotics in the treatment of impetigo contagious. Acta Derm Venereol. 1994;74:460-2.

13. Graham R, Masters-Awatere B. Experiences of Māori of Aotearoa New Zealand's Public Health System: A Systematic Review of Two Decades of Published Qualitative Research. 2020;44.

14. Yadav K, Nath A, Suh KN, Sikora L, Eagles D. Treatment failure definitions for non-purulent skin and soft tissue infections: A systematic review. Infection. 2019:1-9.

15. Statistics New Zealand. 2018 census data. https://www.stats.govt.nz/2018-census/ accessed Sept 2020.

16. Reid P. Achieving health equity in aotearoa: Strengthening Responsiveness to Māori in Health Research. N Z Med J. 2017;130(1465):96-103.

17. Gray S, Lennon D, Anderson P, Stewart J, Farrell E. Nurse-led School-Based Clinics for Skin Infections and Rheumatic Fever Prevention: Results from a pilot study in South Auckland. Journal of the New Zealand Medical Association NZMJ. 2013;126.

18. Anderson P. Nurse-led School-Based Clinics for Rheumatic Fever Prevention and Skin Infection Management: Evaluation of Manakidz programme in Counties Manukau. N Z Med J. 2016;129(1428):37-46.

19. R Core Team. R: A language and environment for statistical computing, $R$ foundation for statistical computing. Vienna, Austria. 2019. https://www.R-project.org/.

20. Bowen AC, Dr, Tong, Steven YC, PhD, Andrews RM, Prof, et al. Short-course oral co-trimoxazole versus intramuscular benzathine benzylpenicillin for impetigo in a highly endemic region: An open-label, randomised, controlled, non-inferiority trial. Lancet The. 2014;384(9960):2132-40.

21. Zhao CY, Hao EY, Oh DD, et al. A comparison study of clinician-rated atopic dermatitis outcome measures for intermediate- to dark-skinned patients. British journal of dermatology (1951). 2017;176(4):985-92.

\section{Figures}




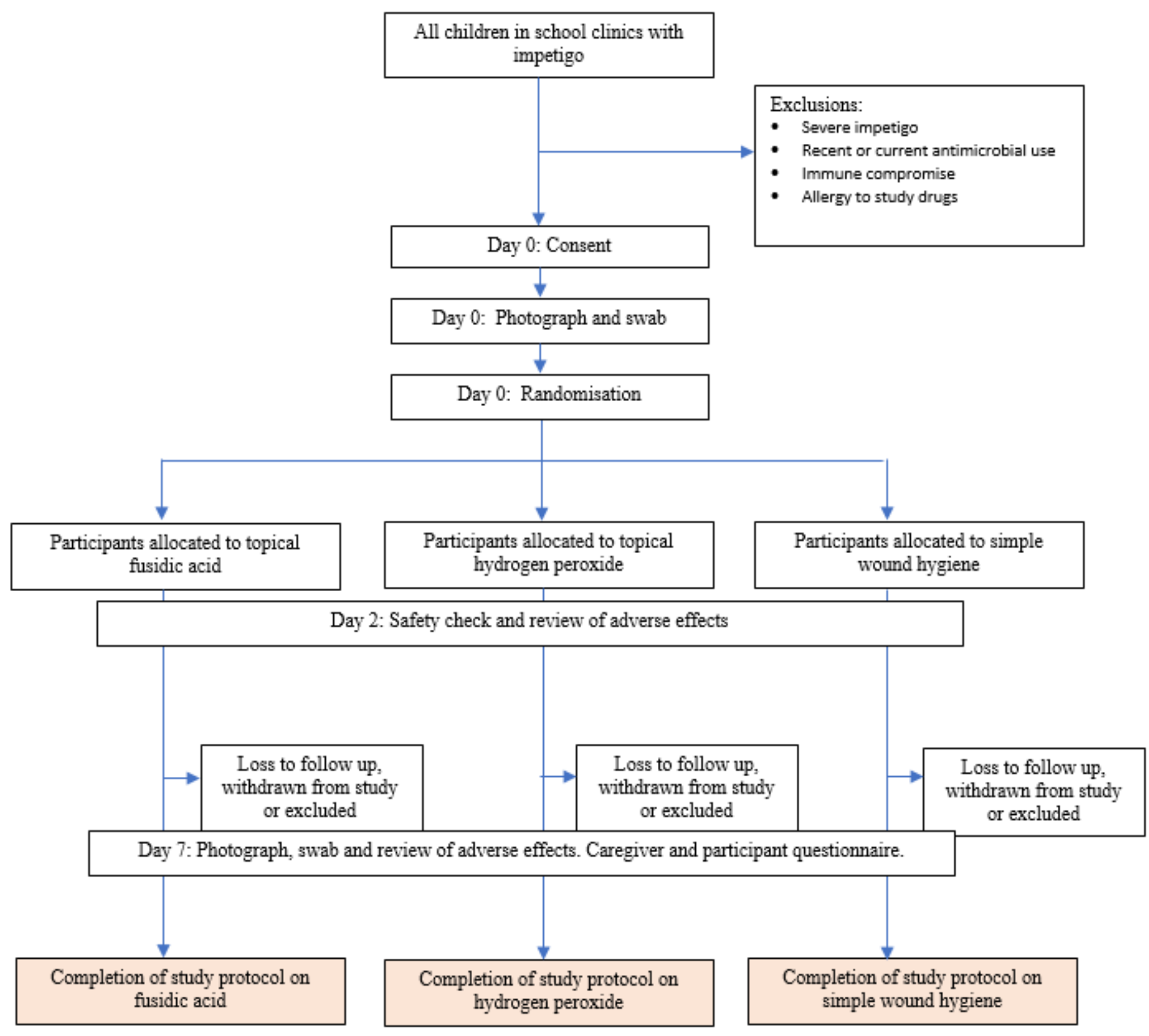

\section{Figure 1}

Schedule of enrolment, interventions, and assessments

\section{Supplementary Files}

This is a list of supplementary files associated with this preprint. Click to download.

- Appendix1.Consentform.pdf

- SPIRITChecklistdownload8Jan133.doc 